\title{
Cultural Adaptivity for the Semantic Web
}

\author{
Katharina Reinecke \\ Department of Informatics, University of Zurich, \\ Binzmühlestr. 14, 8050 Zurich, Switzerland \\ reinecke@ifi.uzh.ch
}

\begin{abstract}
Our research explores the possibilities for factoring culture into user models, working towards cultural adaptivity in the Semantic Web. The aim is to automate the localization process by acquiring details about an individual's cultural background. The paper shows how we have based our approach on research findings from related fields, outlining the connection between the cultural user and domain model ontologies, and a test environment. Furthermore, we describe a preliminary plan of how to validate our cultural user model by comparison to our previous experience in the manual adaptation of imbuto.
\end{abstract}

\section{Motivation}

Culturally adapted user interfaces have been demonstrated to outperform ordinary applications for culturally diverse user groups with regard to user acceptance and usability in general. Our experience with imbuto, a learning software developed for Rwandan agricultural advisers, underlined the assumption that work efficiency and user satisfaction drastically increased with the manual localization of the UI [1]. On the other hand, extensive circular adaptations over five months were required to be implemented on-site in Rwanda. An extension of the target group to encompass other cultures would require even greater time. Cultural software localization can, therefore, be seen as extremely time-consuming and prohibitively expensive. However, this is not the only problem with manual localization: Culture, by its elusive and intangible nature, makes it hard to recognize one's own preferences. Therefore, users cannot be expected to know themselves where the system should be adapted 2].

We propose to tackle these problems with cultural adaptivity, and thus, to automate the personalization process with the help of a cultural user model. After exploring our requirements and aligning them with several techniques for building adaptive systems (see [3), our requirements for a distributed and reusable user profile - enabling an extension to numerous application and devices for cross-system personalization 4] - led to the decision to exploit Semantic Web services for our purposes.

In the following, we discuss the current state of related research (Section 21), present our own advances towards a semantically represented cultural user model and provide a research plan for advancing and evaluating this system (Section 3). The paper closes with a discussion of our contribution to current Semantic Web research and Human-Computer Interaction (HCI) in general.

K. Aberer et al. (Eds.): ISWC/ASWC 2007, LNCS 4825, pp. 946 950, 2007.

(C) Springer-Verlag Berlin Heidelberg 2007 


\section{Related Work}

The idea of semantic user modeling for culturally adaptive systems is based on work from the related fields of international usability and culture, semantic user modeling and adaptive hypermedia. However, these isolated research efforts have not yet brought forward an approach comparable to ours. To our knowledge, Kamentz [5] is alone in her attempt to integrate culture in an approach to modeling learner characteristics of an adaptive e-learning application. The resulting system, however, does not meet our requirement for cross-system personalization, as introduced in 4 .

Culture and Usability. Considering culture in the software development process has been suggested by many researchers in the field of HCI. The motivating factor behind it is the enormous improvements in working efficiency seen as a result of (manually) localized UIs 6 67. Researchers in this area have mainly concentrated on applying measurable classifications of culture developed by cultural theorists Hofstede and Trompenaar [89] to the field of UI design [10/7/5]. In our approach, the cultural dimensions developed by Hofstede serve as a basis for the initial classification of the user model (please refer to [3] for more details).

Semantic User and Domain Modeling. With the rise of the Semantic Web, application specific user models have been countered with approaches to distributed user modeling [11, sharing user models through ontologies 12. The Portal Adaptation Ontology [13, for example, includes a user profile ontology as well as an adaptation ontology that holds knowledge about the adaptation process. Further efforts include the General User Model Ontology (GUMO), which supplies a method "for the uniform interpretation of distributed user models in intelligent Semantic Web enriched environments" [14. Parts of GUMO's user modeling functionalities have been used by the Personal Reader project, which provides an environment for the construction of personalized Web content readers [15]. GUMO also serves as a supplement for our cultural user model.

Apart from the representation of a user model, user modeling requires the acquisition of data throughout the user's interaction with the system. So far, most personalization mechanisms have constructed their user model by analyzing navigational behavior [16. A more detailed approach to tracking user behavior is described in [17]: Here, a semantically rich user model is built by combining the web development technique AJAX with the Semantic Web. Advantages of this approach are the on-the-fly adaptation that removes the need for reloading a page and the ability to record scrolling, mouse-over and keystroke events [17. As we assume that inferring the user's culture does require such a sophisticated navigation analysis, we have adopted this approach for our test application.

Furthermore, we need to perform some kind of inferencing on the obtained data to access implicit information. Efforts in this direction include the Web Usage Ontology Generation [12] that applies fuzzy logic techniques to derive knowledge about the user's behavior. Only recently, Roberto and da Silva [18] presented an algorithm to classify the user's intentions recorded in a semantic 
log. Again, this approach presumes that the analyzed web site has an underlying adaptation ontology. In the past, many projects also made use of the strong reasoning support provided by the Ontobroker inference engine [19].

\section{Research Plan}

The aim of our work is to extend existing approaches to user modeling in the Semantic Web by adding cultural dimensions to the user's profile. The information about the user's position in the cultural dimensions will provide applications in different domains with the ability to adequately localize the UI and its content.

We will integrate our cultural user model in an AJAX portal, which we are currently developing to support the online storage of to-do lists and notes. The application will be accessible to different devices such as mobile phones or computers. The target group consists of people from around the world, however, we will initially test the portal with students from Switzerland (thus being influenced by the French, German, Italian and Swiss cultures) as well as with Rwandan students.

\subsection{Current State of Our Research}

User and Domain Model. So far, we have developed an OWL ontology that is composed of essential classes for outlining the user's culture. This cultural user modeling ontology covers general information, such as the user's birthplace, the religion or the parents' nationality. Each of these classes have been allocated an influence factor. According to the user's age, the duration of stays abroad is also included in the determination of his culture.

Furthermore, the cultural user modeling ontology contains Hofstede's five dimensions (see [8]) which can be extracted to represent the user's cultural position in a five-dimensional space. This information is then linked with the domain model ontology which we are currently working on. Our preliminary work in this respect includes the integration of results from existing evaluations on the effects of culture on UIs and content adaptation. The outcome were guidelines for a cultural domain model that explain the necessary localization strategies for each of the five dimensions. The classification of the user into the dimensions is carried out by taking the information gained from an initial interview about his origin and other cultural influences. Taking the percentages of each influencing factor, we can calculate the user's exact score for each dimension. Our cultural domain model then gives information about the effect on the UI localization: A high score in the dimension Uncertainty Avoidance, for example, triggers the adaptation to a linear navigation and a complex but clearly arranged spatial organisation of elements, among others.

Reasoning. Making inferences about culture works nearly the same way as in conventional personalization systems: The cultural dimensions are represented by integer values in the user modeling ontology and are assigned a default value that describes the average rating. Additionally, we are going to allocate each 
cultural dimension to a certain user behavior. In our approach, this behavior is tracked by an annotated AJAX interface and is stored in the user model. Furthermore, we plan to align the resulting patterns with the underlying adaptation ontology which triggers the adaptation of UI elements.

Application Domain. At present, we are implementing a portal combining Semantic Web and AJAX technologies. From our experience with imbuto we have gained substantial information about the manual localization of a UI and the content to the Rwandan culture. This provides the means for the verification of our cultural user and domain model: If evaluations with Rwandans using the portal produce comparable results regarding user satisfaction and work efficiency, our approach can be assumed to have successfully automized the UI localization.

\subsection{Plan for Further Research}

Although we have laid the foundations for cultural adaptivity, there are still several steps to take: Firstly, we have to revise the user and adaptation ontologies before connecting them to the portal. The adaptation mechanisms have to be extended with adequate inference rules that map certain user behaviour to interface adaptations. Secondly, we plan to validate the ontologies. Evaluations will be carried out in 2008 with Bachelor and Master students from the University of Zurich and at the National University of Rwanda. Each user group has to work with a non-localized version first in order to be able to classify the usability tested after the automatic localization. The evaluations with Rwandan students are especially useful to verify our results: We plan to compare the automatic adaptations resulting from the use by the Rwandan students with our results of the manual adaptation in imbuto. More precisely, the evaluations will include the same usability test procedures as used for imbuto, as well as an analysis of the interface characteristics after the adaptation. These evaluations will then help us to refine our ontologies.

\section{Conclusion}

We presented our approach to cultural adaptivity using the Semantic Web for both user modeling and adaptation. We extend upon research cited throughout this paper in various ways. Firstly, we combine knowledge about cultural localization with personalization techniques. Secondly, we work towards a reusable, distributed user model that allows for the mapping of culture to various application domains. Thirdly, we are implementing a test environment that allows for extensive usability tests in regards to culturally extended ontologies and personalization in general. Therefore, our scientific contribution in the field of personalization in the Semantic Web is an extended user model ontology that we will verify by comparing manual and automatic adaptation to a target culture. The practical impact of our approach is an automatic cultural localization possibility that reduces both development time and expenses. 


\section{References}

1. Reinecke, K.: A Learning Platform for the Education of Agricultural Advisers in Rwanda (2005), http://www.ifi.uzh.ch/ddis/people/reinecke/

2. Kobsa, A.: User Modeling: Recent Work, Prospects and Hazards. In: Adaptive user interfaces: Principles and practice., North-Holland, pp. 111-128 (1993)

3. Reinecke, K., Bernstein, A.: Culturally Adaptive Software: Moving Beyond Internationalization. In: Proceedings of the 12th International Conference on HumanComputer Interaction, Beijing, China, Springer, Heidelberg (2007)

4. Mehta, B., Nejdl, W.: Intelligent Distributed User Modelling: from Semantics to Learning. In: International Workshop on Ubiquitous and Decentralized User Modeling, Corfu, Greece (2007)

5. Kamentz, E.: Adaptivität von hypermedialen Lernsystemen. Phd thesis, University of Hildesheim (2006)

6. Barber, W., Badre, A.: Culturability: The Merging of Culture and Usability. In: Proceedings of the 4th Conference on Human Factors and the Web (1998)

7. Ford, G., Gelderblom, H.: The Effects of Culture on Performance Achieved through the use of Human Computer Interaction. In: Proceedings of the 2003 Annual Research Conference of the South African Institute of Computer Scientists and Information Technologies on Enablement through Technology (SAICSIT) (2003)

8. Hofstede, G.: Culture's Consequences: Comparing values, behaviours and organisations across nations. Sage Publications Inc., Thousand Oaks (2003)

9. Trompenaars, F., Hampden-Turner, C.: Riding the Waves of Culture: Understanding Cultural Diversity in Business. Nicholas Brealey Publishing Ltd (1997)

10. Baumgartner, V., Marcus, A.: A Practical Set of Culture Dimensions for Global User-Interface Development. In: Proceedings of the 6th Asia-Pacific Conference on Computer-Human Interaction (2004)

11. Dolog, P., Nejdl, W.: Challenges and Benefits of the Semantic Web for User Modelling. In: Adaptive Hypermedia 2003 (2003)

12. Zhou, B., et al.: Web Usage Mining for Semantic Web Personalization. In: Workshop on Personalization on the Semantic Web, Edinburgh, UK, pp. 66-72 (2005)

13. Stojanovic, L., Thomas, S.: Fostering Self-Adaptive e-Government Service Improvement Using Semantic Technologies (2006)

14. Heckmann, D., et al.: GUMO - the General User Model Ontology. In: The 10th International Conference on User Modeling, Edinburgh, UK, pp. 428-432. Springer, Heidelberg (2005)

15. Henze, N.: Personalization Services for e-Learning in the Semantic Web. In: Proceedings of the 2nd International Workshop on Adaptive Systems for Web-Based Education: Tools and reusability, Amsterdam, The Netherlands (2005)

16. Eirinaki, M., Vazirgiannis, M.: Web Mining for Web Personalization. ACM Transactions on Internet Technology (TOIT) 3(1), 1-27 (2003)

17. Schmidt, K., et al.: On Enriching Ajax with Semantics: The Web Personalization Use Case. In: Proceedings of the 4th European Semantic Web Conference, Innsbruck, Austria (2007)

18. Roberto, R.L., da Silva, S.: An Approach for Identification of User's Intentions during the Navigation in Semantic Websites. In: Proceedings of the 4th European Semantic Web Conference, Innsbruck, Austria (2007)

19. Oberle, D., Wenke, D., Volz, R., Staab, S.: OntoBroker and OntoEdit Adaptation. Technical report (2003) 